\title{
Patiomed
}

\section{Medizinische Versorgung der besonderen Art}

\begin{abstract}
Ein neuer medizinischer Konzern sorgt für Wirbel. Die neue Aktiengesellschaft, die mit der Gründung von Ärztlichen Versorgungs-
\end{abstract} Zentren eine bestmögliche Patientenversorgung anstrebt, zeichnet sich durch besondere Eigentümerverhältnisse aus.

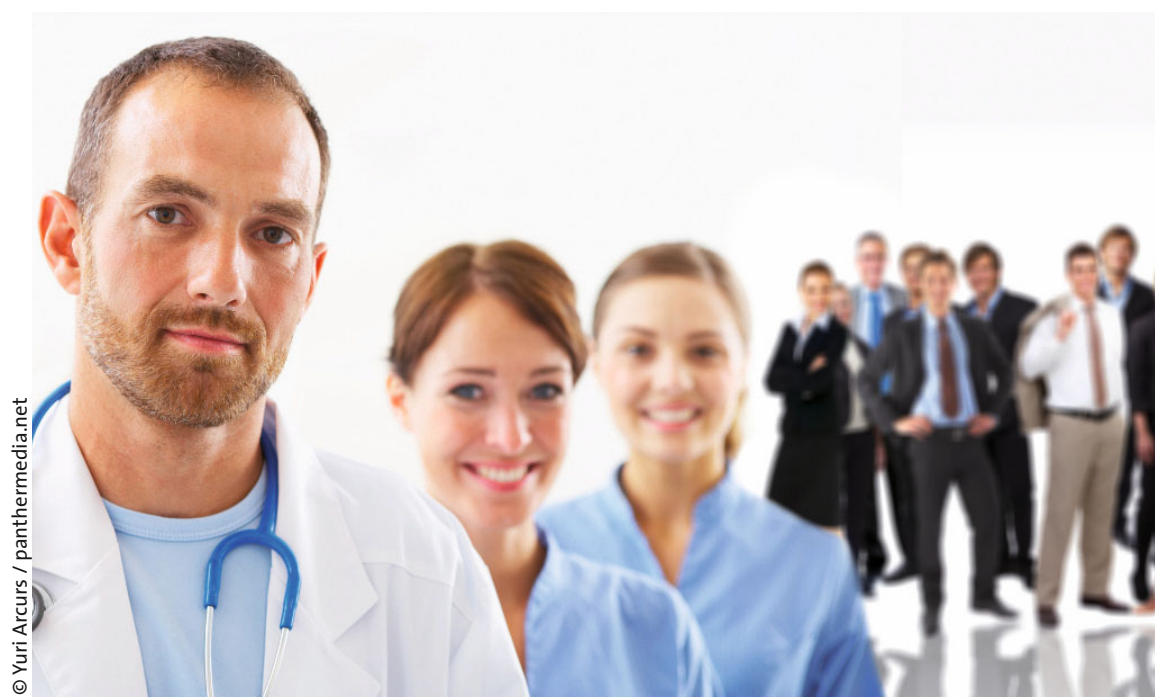

Die Patiomed plant eine „lebenslagenorientierte ärztliche Versorgung“ - interessant ist dabei, wer hinter dem Medizinkonzern steht.

A $\mathrm{m} 1$. Juli ging relativ unbemerkt von der Ärzteschaft ein neuer medizinischer Konzern an den Start, der es sich zum Ziel gesetzt hat, eine innovative Art von medizinischen Versorgungszentren in Deutschland zu betreiben. Die Patiomed möchte „lebenslagenorientierte ärztliche Versorgung“ managen. Das wäre natürlich nur eine neue Facette der medizinischen Versorgung, die durch die neue Gesetzeslage begünstigt wird, wenn es da nicht eine Besonderheit gäbe, die bei sehr vielen Ärzten ein rotes Warnlämpchen aufleuchten lässt: Die Idee und konsequente Verwirklichung der Patiomed AG geht auf die Initiative einzelner Vertreter der kassenärztlichen Vereinigungen der Län- der und des Bundes zurück: Im Jahr 2007 wurde die Äskulapstiftung durch circa 40 Funktionäre der kassenärztlichen Vereinigungen (KV) als Privatpersonen gegründet. Satzungsziel ist unter anderem die Gründung von Medizinischen Versorgungszentren.

Circa ein Jahr später wurde dann für das operative Geschäft die KVmed $\mathrm{GmbH}$ gegründet mit einer Beteiligungsgesellschaft, die inzwischen unter anderem die Asklepioskliniken mit in dieses Firmenkonglomerat einbezieht. Der bis dato letzte Schritt in Richtung eines Medizinkonzerns ist die Gründung der $\mathrm{Pa}$ tiomed AG, an der nun neben der KVmed $\mathrm{GmbH}$ auch die deutsche Ärzteund Apothekerbank, der deutsche Ärzte- verlag und eine Schweizer Kapitalgesellschaft beteiligt sind.

Schon diese Konstellation hat für so manchen Arzt einen strengen Beigeschmack. Wie passt es zusammen, dass die Vertreter der Mitglieder der KVen gleichzeitig als Privatiers in solch ein Konstrukt involviert sind, dass ja durchaus dazu geeignet ist, alteingesessenen Praxen das Leben schwer zu machen? Bedenkt man in diesem Fall auch noch, dass die Bedarfsplanung durch die KV erfolgt (hierzu gibt es in der „kleinräumigen Versorgungsanalyse" einen neuen Vorschlag, welcher der Patiomed durchaus in die Hand arbeiten könnte) und die KVen auch Einblicke in die Einkommenssituation der Kollegen haben, wird klar, woher diese Ängste stammen.

Die Patiomed AG versucht immer wieder klarzustellen, dass sie keine Konkurrenzsituation mit den niedergelassenen Kollegen anstrebe und - ungewöhnlich für eine Aktiengesellschaft auch keine eigentliche Gewinnabsicht habe. Allerdings ist nun ein Businessplan der Patiomed (früher auch „New Company" genannt) aufgetaucht, der in eine andere Richtung deutet und auch darauf hinweist, dass vor allem „gute Marktzugangsmöglichkeiten durch Know-how der KV und Kenntnisse der örtlichen Versorgungssituationen "bestünden. $\mathrm{Ob}$ hier nun ein Interessenskonflikt zwischen der Tätigkeit der KV-Funktionäre als Vertreter der Ärzte mit dem Gewinnstreben als Beteiligte an einem „Medizinkonzern" besteht, mag jeder Kollege selbst entscheiden.

\section{Dr. med. Ilka M. Enger}

Bayerischer Facharztverband 